important observation that rift propagation can occur at the full range of spreading rates. A curious property of rift propagation which I have noticed in my own work is that the long-term rate of propagation is often approximately $50-100$ per cent of the full spreading rate of the ridge, regardless of the spreading rate. The new South Atlantic studies lend further support to this yet-to-be-explained observation.

Ken C. Macdonald is in the Department of Geological Sciences and the Marine Science Institute, University of California, Santa Barbara, California 93106, USA.

\title{
How efficient can you get?
}

\section{Mario Capecchi}

IN the tropics, trypanosomatid protozoa cause numerous severe human diseases, including African sleeping sickness, Chagas' disease and kala-azar disease. Many genes have been identified in these protozoa and for some species there are detailed descriptions of the mechanisms of antigen variation and RNA processing. But little is known about the biological function of the cloned genes. This is mainly because of the difficulty of carrying out genetic analysis in these organisms, which have complex biological cycles involving more than one host and which, when propagated in cell culture, do not exhibit a sexual cycle.

A solution to this problem has just been achieved. On pages 171 and 174 of this issue, Cruz and Beverley ${ }^{1}$, and ten Asbroek, Ouellete and Borst ${ }^{2}$, report on targeted gene replacement in Leishmania major and Trypanosoma brucei, respectively. The high efficiency of targeted mutagenesis found by both groups makes it feasible to inactivate both copies of any chosen gene in these diploid organisms. The technology should prove valuable not only for defining gene function but also for combating these organisms in the fight against the diseases they cause. For example, creating truly attenuated strains of Leishmania should prove to be useful for the development of vaccines. Even for trypanosomes that exhibit antigen variation, such an approach might prove helpful - in these cases, by concentrating on attenuated strains of metacyclic variants, which are the first to colonize the blood and represent only a subset of the total antigen repertoire, clinically applicable vaccines might be obtained.

After the introduction by electroporation of a targeting vector containing a neomycin resistance gene $\left(\right.$ neo $\left.^{t}\right)$ flanked by dihydrofolate reductase thymidylate synthase sequences (dhfr-ts), Cruz and Beverley found that 45 to almost 100 per cent of the G418-resistant Leishmania colonies contained the predicted homologous replacement of the endogenous dhfr-ts sequence with the exogenous sequence. Similarly, ten Asbroek et al. targeted the neo gene into the endogenous tubulin gene cluster. What I find particularly intriguing is the very high efficiency of these homologous recombination events.

In simple eukaryotes such as yeast ${ }^{3}$, and now Leishmania major and Trypanosoma brucei, homologous recombination can predominate over nonhomologous recombination. In organisms of slightly greater complexity, Dictyostelium for example, the ratio of homologous to nonhomologous recombination events, following the introduction of exogenous DNA, is roughly 1:5 (ref. 4). In mamma$a$
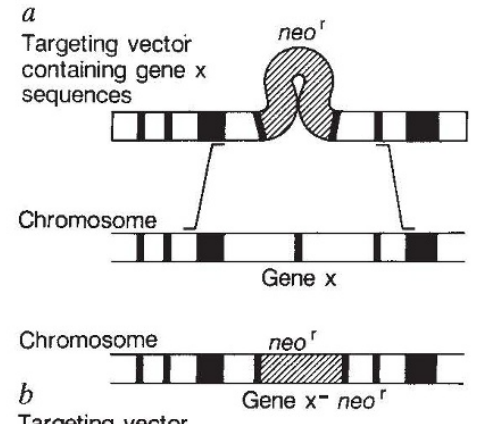

Targeting vector

containing gene $x$

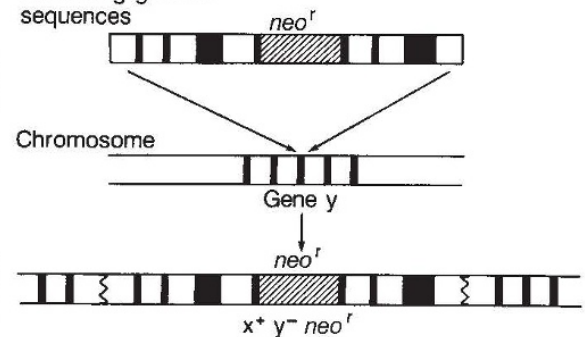

a, Homologous recombination (gene targeting). The targeting vector, a neomycin resistance gene (neo') flanked by gene $x$ sequences is shown pairing with a chromosomal copy of gene $x$. Homologous recombination between the targeting vector and genomic $x$ DNA results in the specific disruption of the chromosomal copy of gene $x$ (that is, $x^{-}$but now $n e o^{r}$ ). $b$, Nonhomologous recombination (random integration). In nonhomologous recombination the targeting vector is randomly inserted into the chromosomal DNA (here arbitrarily shown to be inserted into gene $y$ ). The cells are still $\mathrm{x}^{+}$because the targeting vector was inserted somewhere else in the genome. Most nonhomologous or random insertions of the targeting vector occur through the ends of the targeting vector, presumably at an existing double-strand break in the chromosomal DNA. The solid boxes represent exons, the open boxes introns. lian cells this ratio is about 1:1,000 (ref. 5). This shift in the ratio does not represent a greater efficiency of homologous recombination in simple eukaryotes but rather an increase in the efficiency of nonhomologous or illegitimate recombination in more complex organisms.

Why have complex organisms developed such an efficient machinery for mediating nonhomologous recombination? Although it is always dangerous in science to approach questions of "why" rather than 'how', an answer might reside in the relative investment of the species in creation of an individual. Both types of somatic recombination process, homologous and nonhomologous, are part of the repair machinery used by all organisms to counteract the continual damage to DNA resulting from exposure to chemicals and radiation. A double-strand break in the DNA, induced by such agents, results in the loss of all genes distal to the centromere. In single-cell eukaryotes, such a break might result in the death of the cell. But relative to a complex organism, little has been invested in the creation of a single-cell individual.

A similar double-strand break in the DNA of a cell from a complex organism, if it occurs relatively early during embryogenesis, might also result in the loss of the individual. But here the investment is much greater. Under these circumstances, a strategy to save the individual might be to jam the pieces of DNA together by nonhomologous recombination thereby preventing the loss of distal genes. Homologous recombination, in using the information from the other allele, could then be used to repair the damage at the newly formed junction. Compounding the problem faced by more complex organisms is the higher probability of acquiring deleterious double-strand breaks in DNA as a consequence of their having larger genomes.

With these latest successes of gene targeting in Leishmania and Trypanosoma, there are now even greater incentives to develop the technology in organisms of intermediate complexity such as Caenorhabditis elegans and Drosophila. The ratio of homologous to nonhomologous recombination in these organisms might prove to be greater than in mammalian cells, thereby helping in the application of gene targeting to certain kinds of genetic analysis.

Mario Capecchi is in the Department of Biology, University of Utah, 201 Biology Building, Salt Lake City, Utah 84112, USA.

1. Cruz, A. \& Beverley, S.M. Nature 348, 171-173(1990) 2. ten Asbroek, A.L.M.A., Ouellete, M. \& Borst, P. Nature 348, 174-175 (1990).

. Hin 174 A., Hicks, J. \& Fink, G.R. Proc. natn. Acad. SCi. U.S.A. 75, 1929-1933 (1978)

4. De Lozanne, A. \& Spudich, J.A. Science 236, 1086-1091 (1987).

5. Thomas, K.R. \& Capecchi, M.R. Cell 52, 503 -512 (1987). 